MTX (3), HCQ (1), AZA (1) MMF (1). A significant improvement of the dyspnea was observed. FVC and HRCT showed an improvement in the period between 6 and 12 months. DLCO remained stable in the majority of the patients (\%). DAS28 also improved.

After a follow-up of 12 months, the only serious adverse effect was a severe Infection respiratory.

Table 1

\begin{tabular}{lcccc}
\hline & Baseline & 3 months & 6 months & 12 months \\
\hline MMRC, $n$ (\%) & 18 & 15 & 16 & 16 \\
- No change & & $10(67)$ & $10(63)$ & $10(63)$ \\
- Improvement & & $5(33)$ & $6(37)$ & $6(37)$ \\
- Worsening & & 0 & 0 & 0 \\
CVF, n (\%) & 16 & 10 & 5 & 13 \\
- No change & & $7(70)$ & $5(100)$ & $10(77)$ \\
- Improvement & & $1(10)$ & 0 & $3(23)$ \\
- Worsening & & $2(20)$ & 0 & 0 \\
DLCO, n (\%) & 14 & 6 & 3 & 12 \\
- No change & & $5(83)$ & $1(33)$ & $8(66)$ \\
- Improvement & & 0 & $2(67) 72(17)$ & \\
- Worsening & & $1(17)$ & 0 & $2(17)$ \\
HRCT, n (\%) & & 7 & 9 & 7 \\
- No change & & $6(86)$ & $7(78)$ & $4(57)$ \\
- Improvement & & $1(14)$ & $1(11)$ & $3(43)$ \\
- Worsening & & 0 & $1(11)$ & 0 \\
JOINT, DAS28 - Mean & $4.32 \pm 1.35$ & $3,21 \pm 0.73$ & $3,44 \pm 0.87$ & $2.83 \pm 0.70$ \\
CRP (mg/dl)-, Mean & $2.01 \pm 2.35$ & $1.12 \pm 0.9$ & $1.98 \pm 1.95$ & $1.03 \pm 0.9$ \\
ESR (mm/1sth), Mean & $49.0 \pm 30.64$ & $37.79 \pm 37,73$ & $38.63 \pm 30.63$ & $24.50 \pm 22$ \\
\hline & & & & \\
& & & & \\
& & & &
\end{tabular}

Conclusions: RTX seems to be an effective and relatively safe treatment in RA patients with ILD. However, these data should be verified in prospective and randomized studies.

Disclosure of Interest: None declared

DOI: 10.1136/annrheumdis-2017-eular.3275

\section{FRI0227 SARILUMAB SIGNIFICANTLY SUPPRESSES CIRCULATING BIOMARKERS OF BONE RESORPTION AND CARDIOVASCULAR RISK COMPARED WITH ADALIMUMAB: BIOMARKER ANALYSIS FROM THE PHASE 3 MONARCH STUDY}

C. Gabay ${ }^{1}$, J. Msihid ${ }^{2}$, C. Paccard ${ }^{2}$, M. Zilberstein ${ }^{3}$, N.M. Graham ${ }^{4}$, A. Boyapati 4 . ${ }^{1}$ University Hospitals of Geneva, Geneva, Switzerland; ${ }^{2}$ Sanofi R\&D, Chilly-Mazarin, France; ${ }^{3}$ Sanofi R\&D, Bridgewater; ${ }^{4}$ Regeneron Pharmaceuticals, Inc, Tarrytown, United States

Background: MONARCH (NCT02332590) was a randomized, active-controlled, double-blind, double-dummy, phase 3 superiority trial comparing sarilumab monotherapy with adalimumab monotherapy. Exploratory biomarkers associated with inflammation, bone erosion, and cardiovascular (CV) risk were evaluated in this study.

Objectives: To compare the effects of sarilumab monotherapy vs adalimumab monotherapy on circulating biomarkers associated with acute-phase response (CRP, serum amyloid A [SAA]), bone resorption (RANKL and osteoprotegerin [OPG]), and CV risk (lipoprotein (a) [Lp(a)]) in patients from MONARCH.

Methods: Sera were analyzed at baseline and posttreatment through wk 24 from patients who consented to biomarker analyses and received SC sarilumab $200 \mathrm{mg} \mathrm{q} 2 \mathrm{w}(\mathrm{N}=153)$ or adalimumab $40 \mathrm{mg}$ q2w $(\mathrm{N}=154)$. Biomarkers were assessed using validated ELISAs. Nonparametric methods were used to evaluate differences in the percent change from baseline in biomarker levels between treatments at each time point. Percent change from baseline in biomarkers at wk 24 was also compared, separately by treatment group, between ACR50 responders and nonresponders at wk 24. The Benjamini-Hochberg procedure was used to correct $P$ values for multiplicity and control false discovery rate. Significance level was $P<0.05$.

Results: A significant difference in RANKL was observed at wks 2 and 24 between sarilumab and adalimumab groups $(P<0.0001$; Table). Numerically, RANKL decreased after sarilumab and increased after adalimumab treatment. Significantly greater reductions in $\mathrm{Lp}(\mathrm{a}), \mathrm{SAA}$, and CRP were observed at wks 12 and 24 after treatment with sarilumab vs adalimumab. The difference in OPG between groups was significant at wk 2 only.

Table 1. Median Percent Change From Baseline in Serum Concentrations of Circulating Biomarkers

\begin{tabular}{llcc}
\hline & & Sarilumab 200 mg q2w $(\mathrm{N}=153)$ & Adalimumab 40 mg q2w $(\mathrm{N}=154)$ \\
\hline CRP & Wk 12 & $-94.2^{\dagger}$ & -29.7 \\
& Wk 24 & $-94.0^{\dagger}$ & -24.0 \\
SAA & Wk 12 & $-79.9^{\dagger}$ & -32.2 \\
& Wk 24 & $-83.2^{\dagger}$ & -17.4 \\
RANKL & Wk 2 & $-2.5^{\dagger}$ & 4.4 \\
& Wk 24 & $-18.3^{\dagger}$ & 10.5 \\
OPG & Wk 2 & $0.9^{\star}$ & -4.0 \\
& Wk 24 & 2.2 & 3.3 \\
Lp(a) & Wk 12 & $-35.0^{\dagger}$ & -0.4 \\
& Wk 24 & $-41.0^{\dagger}$ & -2.8 \\
\hline
\end{tabular}

${ }^{\star}$ Adjusted $P<0.01$ vs adalimumab. ${ }^{\dagger}$ Adjusted $P<0.0001$ vs adalimumab.
At wk 24, change in OPG, RANKL, and Lp(a) did not differ between ACR50 responders and nonresponders at wk 24 in either treatment group. Responders in both groups demonstrated greater reductions vs nonresponders in CRP (sarilumab, $-95.8 \%$ vs $-87.3 \%$; adalimumab, $-47.6 \%$ vs $-6.4 \%$; all $P<0.01$ ); a trend was observed for SAA (sarilumab, $-92.3 \%$ vs $-73.2 \%$; adalimumab, $-33.0 \%$ vs $0.0 \%$; unadjusted $P<0.05$, not significant after adjustment).

Conclusions: Sarilumab monotherapy significantly suppressed bone-resorptive and $\mathrm{CV}$ risk markers to a greater degree than adalimumab monotherapy. Reductions in CRP were significantly different in ACR50 responders vs nonresponders after either treatment. Analyses to assess predictive and prognostic effects of biomarkers (including markers of myeloid and lymphoid synovial phenotypes) are ongoing.

Acknowledgements: This study was sponsored by Sanofi Genzyme and Regeneron Pharmaceuticals, Inc. Editorial support was provided by MedThink SciCom and funded by Sanofi Genzyme and Regeneron Pharmaceuticals, Inc.

Disclosure of Interest: C. Gabay Consultant for: Roche, Merck, AbbVie, Pfizer, Bristol-Myers Squibb, Sanofi-Aventis, and AB2 Bio, J. Msihid Shareholder of: Sanofi R\&D, Employee of: Sanofi R\&D, C. Paccard Shareholder of: Sanofi R\&D, Employee of: Sanofi R\&D, M. Zilberstein Shareholder of: Sanofi R\&D, Employee of: Sanofi R\&D, N. Graham Shareholder of: Regeneron Pharmaceuticals, Inc, Employee of: Regeneron Pharmaceuticals, Inc, A. Boyapati Shareholder of: Regeneron Pharmaceuticals, Inc, Employee of: Regeneron Pharmaceuticals, Inc DOI: 10.1136/annrheumdis-2017-eular.4534

\section{FRI0228 UGT1A1 GENETIC VARIANTS ARE ASSOCIATED WITH INCREASES IN BILIRUBIN LEVELS IN RHEUMATOID ARTHRITIS PATIENTS TREATED WITH SARILUMAB}

A. Damask ${ }^{1}$, A. Boyapati ${ }^{1}$, J.D. Hamilton ${ }^{1}$, S. Hamon ${ }^{1}$, C. Paccard ${ }^{2}$, J. Parrino ${ }^{1}$, J. van Adelsberg ${ }^{1}$, N.M. Graham ${ }^{1}$, J. Penn ${ }^{1}$, A. Lopez ${ }^{1}$, J. Reid ${ }^{1}$, J. Overton ${ }^{1}$,

A. Baras ${ }^{1}$, A.R. Shuldiner ${ }^{1}$, C. Paulding ${ }^{1} .{ }^{1}$ Regeneron Pharmaceuticals, Inc, Tarrytown, United States; ${ }^{2}$ Sanofi R\&D, Chilly-Mazarin, France

Background: Sarilumab is a human mAb that blocks IL-6 from binding to both membrane-bound and soluble IL-6R $\alpha$. Variants in the UGT1A1 gene have been shown to be strongly associated with increased unconjugated bilirubin levels in patients treated with tocilizumab, another IL-6R $\alpha$ inhibitor. ${ }^{1,2}$ UGT1A1 encodes the enzyme responsible for the glucuronidation of bilirubin and variation in this gene is also responsible for Gilbert's syndrome, a mild benign condition characterized by elevations in unconjugated bilirubin and jaundice. The underlying main genetic variation responsible for Gilbert's syndrome has been identified as a TA repeat located in the promoter of UGT1A1 (UGT1A1*28 allele), which is in linkage disequilibrium with a single nucleotide polymorphism, rs6742078, previously associated with higher bilirubin levels after tocilizumab treatment. ${ }^{1}$

Objectives: To test for an association between rs6742078 and bilirubin levels in RA patients treated with sarilumab.

Methods: DNA was collected from patients enrolled in MOBILITY (NCT01061736), which evaluated the efficacy and safety of sarilumab + methotrexate (MTX) in RA patients with inadequate response to MTX. The pharmacogenetic analysis was conducted in 599 Caucasian patients treated with MTX + sarilumab (150 or $200 \mathrm{mg} \mathrm{q2w}$ ) or placebo. Log-transformed unconjugated and total bilirubin levels were analyzed at baseline and over the treatment period (using maximum bilirubin).

Results: There was a strong association between the rs6742078 TT genotype and higher unconjugated bilirubin levels. The least squares mean (SE) for patients at baseline with the TT genotype was $0.48(0.02) \mathrm{mg} / \mathrm{dL}$ vs $0.25(0.009)$ and 0.21 $(0.009) \mathrm{mg} / \mathrm{dL}$ for those with GT and GG genotypes, respectively $\left(p=1.02 \times 10^{-21}\right)$. After sarilumab treatment, the difference between genotype groups increased over the course of the study $\left(p=4.3 \times 10^{-10} ;\right.$ Figure). In the binary analysis of maximum total bilirubin in sarilumab-treated patients, the TT genotype was significantly associated with mild bilirubin elevations ( $\mathrm{OR}=34.7 ; p=1.2 \times 10^{-8}$; Table).

Table 1. Maximum Total Bilirubin in Sarilumab-Treated Patients by rs6742078 Genotype

\begin{tabular}{lccc}
\hline & \multicolumn{3}{c}{ Maximum total bilirubin, $\mathrm{n}(\%)$} \\
\cline { 2 - 4 } Genotype, $\mathrm{n}(\%)$ & $\leq 1.5 \times \mathrm{ULN}$ & $>1.5 \times \mathrm{ULN}$ & Total \\
\hline GG/GT & $352(92)$ & $4(27)$ & $356(90)$ \\
TT & $29(8)$ & $11(73)^{\mathrm{a}}$ & $40(10)$ \\
& 381 & 15 & 396
\end{tabular}

$\mathrm{OR}=34.7^{\mathrm{b}} ; p=1.2 \times 10^{-8} .{ }^{\text {a Elevations remained } \leq 2 \times \mathrm{ULN} \text {. }{ }^{\mathrm{b}} \text { Logistic regression with recessive ge- }}$ netic model, adjusting for ancestry covariates.

Conclusions: The association observed between the rs6742078 TT genotype in UGT1A1 and unconjugated bilirubin elevations in sarilumab-treated patients is consistent with previous observations in tocilizumab-treated patients. These findings suggest that sarilumab-related increases in bilirubin levels are likely benign and caused by common genetic variation in UGT1A1 and are not due to underlying liver injury.

References:

[1] Mori et al. Mod Rheumatol. 2012;22:515-523.

[2] Lee et al. Pharmacogenet Genomics. 2011;21:365-374

Acknowledgements: This study was sponsored by Sanofi Genzyme and Regeneron Pharmaceuticals, Inc. Editorial support was provided by MedThink SciCom and funded by Sanofi Genzyme and Regeneron Pharmaceuticals, Inc. 

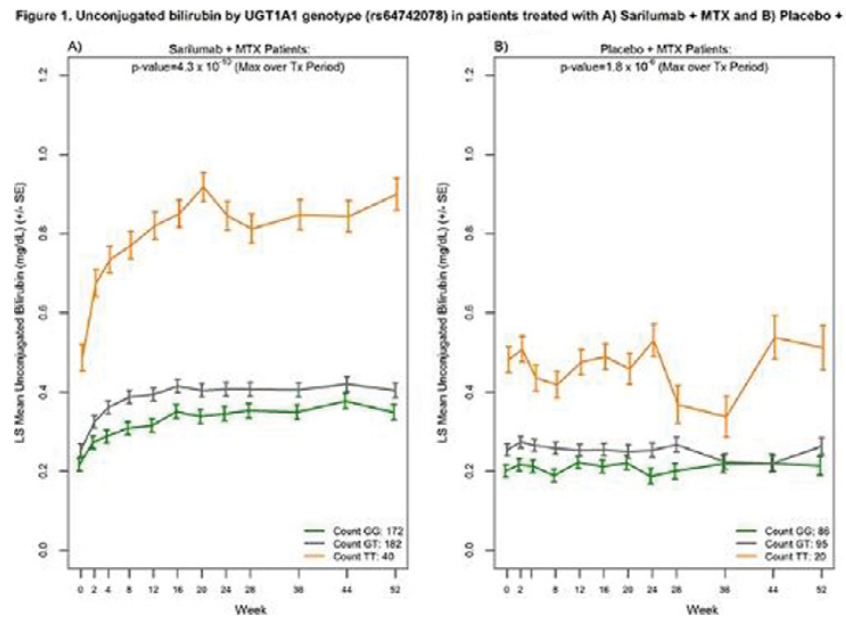

Disclosure of Interest: A. Damask Shareholder of: Regeneron Pharmaceuticals, Inc, Employee of: Regeneron Pharmaceuticals, Inc, A. Boyapati Shareholder of: Regeneron Pharmaceuticals, Inc, Employee of: Regeneron Pharmaceuticals, Inc, J. Hamilton Shareholder of: Regeneron Pharmaceuticals, Inc, Employee of: Regeneron Pharmaceuticals, Inc, S. Hamon Shareholder of: Regeneron Pharmaceuticals, Inc, Employee of: Regeneron Pharmaceuticals, Inc, C. Paccard Shareholder of: Sanofi R\&D, Employee of: Sanofi R\&D, J. Parrino Shareholder of: Regeneron Pharmaceuticals, Inc, Employee of: Regeneron Pharmaceuticals, Inc, J. van Adelsberg Shareholder of: Regeneron Pharmaceuticals, Inc, Employee of: Regeneron Pharmaceuticals, Inc, N. Graham Shareholder of: Regeneron Pharmaceuticals, Inc, Employee of: Regeneron Pharmaceuticals, Inc, J. Penn Shareholder of: Regeneron Pharmaceuticals, Inc, Employee of: Regeneron Pharmaceuticals, Inc, A. Lopez Shareholder of: Regeneron Pharmaceuticals, Inc, Employee of: Regeneron Pharmaceuticals, Inc, J. Reid Shareholder of: Regeneron Pharmaceuticals, Inc, Employee of: Regeneron Pharmaceuticals, Inc, J. Overton Shareholder of: Regeneron Pharmaceuticals, Inc, Employee of: Regeneron Pharmaceuticals, Inc, A. Baras Shareholder of: Regeneron Pharmaceuticals, Inc, Employee of: Regeneron Pharmaceuticals, Inc, A. Shuldiner Shareholder of: Regeneron Pharmaceuticals, Inc, Employee of: Regeneron Pharmaceuticals, Inc, C. Paulding Shareholder of: Regeneron Pharmaceuticals, Inc, Employee of: Regeneron Pharmaceuticals, Inc

DOI: 10.1136/annrheumdis-2017-eular.5016

\section{FRI0229 SURVEY ON TRANSPORTATION AND STORAGE OF BIOLOGICAL THERAPIES BY PATIENTS}

M. Arias Saavedra, C. Aimo, J.J. Astudillo Andrade, D. Alvarez, G. Sequeira, E.M. Kerzberg. Servicio de Reumatologia, Hospital J. M. Ramos Mejia, Buenos Aires, Argentina

Background: in order to ensure the pharmacological action of thermolabile drugs, it is mandatory to keep the cold chain unbroken from manufacturing to administration since lack of observance of these rules may compromise their efficacy. $(1,2)$

Objectives: to ascertain key aspects of transportation and storage of Biological Therapy (BT) on the part of the patients.

Methods: survey among outpatients who either were treated in the centre or attended the centre for the administration of a BT. Inclusion criteria encompassed patients of over 18 years of age, who were receiving a BT (at least one dose over the last 12 months) whose patient information leaflet indicated, "... must be refrigerated at $2^{\circ} \mathrm{C}$ to $8^{\circ} \mathrm{C}\left(36^{\circ} \mathrm{F}\right.$ to $\left.46^{\circ} \mathrm{F}\right)$. Do not freeze." The survey comprised 31 questions about transportation and storage of the BT from the moment of collection from the pharmacy to its administration. This study was approved by the Bioethics Committee. Every patient signed an informed consent form.

Results: Eighty-three patients were interviewed (76\% female and $24 \%$ males), their average age was 53 years old, $60 \%$ had Rheumatoid Arthritis, $24 \%$ Psoriatic Arthritis, and $16 \%$ sustained other conditions. Sixty-four percent were undergoing BT for the first time. Forty-eight percent were enrolled in a patient assistance programme.

Had patients been fully informed of the importance of proper transportation and storage of the drug? No, $20 \%$ acknowledged that the first time they dealt with a $\mathrm{BT}$, they had not been made aware of the relevance of its correct preservation. Are patients administered the BT immediately after collecting it from the pharmacy? No, $77 \%$ collected the drug at least 7 days before administration and though they received the BT in a cooler with ice packs (except in 5 cases) $71 \%$ was uninformed of how long it could be kept in this condition.

Is the BT duly stored in the patient's refrigerator? No, $28 \%$ misplaced the drug ( $65 \%$ in the freezer), and $90 \%$ did not know the temperature range at which it should be kept in the fridge. Only 1 patient used a thermometer to control the appliance temperature.

Is there another crucial point as to BT storage at home? Yes, $53 \%$ of the 83 patients had experienced frequent power cuts in summer; out of these, $59 \%$ on a weekly basis. Forty percent of the 83 patients had power cuts of over 12 hours, $28 \%$ of over 24 hours, and $22 \%$ of over 48 hours. Out of the 83 patients, $37 \%$ took the BT to another house to avoid wasting it, and 4 had to dispose of it due to a protracted power cut. Five patients were away from home for over 48 hours during which the BT was in the fridge and they had not assigned another person to take care of the drug during their absence.

Is the BT removed from the fridge only for its immediate administration? No, $46 \%$ of the respondents travelled to a centre, half of them for over an hour, carrying the BT.

Conclusions: before administering a BT, it is imperative to brief patients on suitable transportation and storage methods. Thus, treatment failure should prompt a thorough assessment of transportation and storage conditions.

\section{References:}

[1] Allegra JR, Brennan J, Lanier V, Lavery R, Markenzie B. Storage temperatures of out-of-hospital medications. Acad Emerg Med. 1999;6:1098-103.

[2] Marco JL, Cuéllar MJ, Pérez-Castelló I, Castelló A, Muelas J, Pelegrí J. La cadena del frío de los medicamentos termolábiles en el domicilio del paciente. Pharm Care Esp. 2008;10:40-3.

Disclosure of Interest: None declared

DOI: 10.1136/annrheumdis-2017-eular.4150

\section{FRI0230 RETENTION RATES OF TNF INHIBITORS AND ABATACEPT USED AS A FIRST BIOLOGIC DMARD IN THE TREATMENT OF RHEUMATOID ARTHRITIS: 8 YEARS OF EXPERIENCE FROM THE RHUMADATA ${ }^{\circledR}$ REGISTRY}

D. Choquette $^{1}$, L. Bessette ${ }^{2}$, E. Alemao ${ }^{3}$, B. Haraoui ${ }^{1}$, F. Massicotte ${ }^{1}$, M. Mtibaa ${ }^{4}$,E. Muratti ${ }^{4}$, J.-P. Pelletier ${ }^{1}$, R. Postema ${ }^{5}$, J.-P. Raynauld ${ }^{1}$,' M.-A. Rémillard ${ }^{1}$, D. Sauvageau ${ }^{1}$, A. Turcotte ${ }^{2}$, É. Villeneuve ${ }^{1}$, L. Coupal ${ }^{1}$ ${ }^{1}$ Rheumatology, Institut de recherche en rhumatologie de Montréal (IRRM), Montréal; ${ }^{2}$ Rheumatology, Centre d'ostéoporose et de rhumatologie de Québec (CORQ), Québec, Canada; ${ }^{3}$ Bristol-Myers Squibb, Princeton, United States; ${ }^{4}$ Bristol-Myers Squibb, Montréal, Canada; ${ }^{5}$ Bristol-Myers Squibb, Uxbridge, United Kingdom

Background: Sustainability of treatment is important to consider when selecting a therapy for chronic conditions such as RA. Sustainability is a useful clinical marker for both long-term efficacy and safety. A recently published randomized controlled trial has demonstrated similar efficacy and safety profiles between abatacept (ABA) and adalimumab over 2 years. ${ }^{1,2}$

Objectives: To assess the long-term sustainability of $A B A$ and anti-TNFs following treatment failure with a conventional synthetic DMARD (cSDMARD) in comparable cohorts of patients (pts) with RA.

Methods: Data from pts with RA seen at two tertiary centres and prescribed either ABA or a TNF inhibitor (adalimumab, certolizumab, etanercept, golimumab or infliximab) as their first biologic (b)DMARD after 1 January 2006 were extracted from the RHUMADATA ${ }^{\circledR}$ registry. The choice of therapy was a joint decision between the pt and the treating physician. Pts were followed until either they discontinued treatment, were lost to follow-up or the cut-off date of 9 January 2017. Pt baseline characteristics were compared using descriptive statistics and the cumulative incidence of biologic agent discontinuation using Kaplan-Meier methods. Overall differences in the discontinuation rates of biologic agents were tested using the log-rank test.

Results: Overall, 641 pts met study criteria; 82 pts received ABA and 559 TNF inhibitors (adalimumab $=136$, certolizumab $=52$, etanercept $=226$, golimumab $=88$ and infliximab=57) as first-line treatment following inadequate response to csDMARDs. No clinically significant differences in baseline characteristics were noted between treatment groups. Most pts were diagnosed after January 2000 $(72.5 \%)$ and were women $(77.5 \%)$. Average age at diagnosis was $47.1 \quad(\mathrm{SD}=13.4)$ years, with a mean disease duration of $7.2(7.8)$ years, and a mean CDAl of 43.1 (32.5) at baseline. No significant differences in retention rates were observed in the ABA and anti-TNF groups (Table, Figure). On average, pts treated with anti-TNFs and ABA maintained their treatment for 1.59 (1.91) and $1.90(2.08)$ years, respectively. Lack of efficacy $(47.6 \%)$ and adverse effects $(22.0 \%)$ were the most commonly cited reasons for treatment discontinuation.

Table 1. Retention of the first bDMARD

\begin{tabular}{lcc}
\hline & TNFi & Abatacept \\
\hline Biologic retention probability at ${ }^{\star}:$ & & \\
12 Months & $71.66 \%(1.92 \%)$ & $70.71 \%(5.15 \%)$ \\
24 Months & $59.85 \%(2.11 \%)$ & $62.16 \%(5.60 \%)$ \\
60 Months & $44.28 \%(2.26 \%)$ & $48.37 \%(6.75 \%)$ \\
96 Months & $35.29 \%(2.46 \%)$ & $41.17 \%(7.43 \%)$ \\
bDMARD retention time (years) & $4.83(0.18)$ & \\
Mean (SE) & $0.86(0.72-1.00)$ & $0.71(0.45)$ \\
Lower quartile, $(95 \% \mathrm{Cl})$ & $3.83(2.87-4.67)$ & $0.86(0.50-1.24)$ \\
Median, $(95 \% \mathrm{Cl})$ & & $4.53(2.34-++)$ \\
\hline
\end{tabular}

*\% (\% standard error) survival.

Conclusions: Abatacept and TNF inhibitors demonstrate similar sustainability at 8-year, supporting studies ${ }^{1,2}$ that demonstrate that abatacept used after csDMARDs inadequate response is as safe and effective as a TNF targeting agents in the long term. 